\title{
SHAPE PROPERTIES OF WHITNEY MAPS FOR HYPERSPACES
}

\author{
HISAO KATO
}

\begin{abstract}
In this paper, some shape properties of Whitney maps for hyperspaces are investigated. In particular, the following are proved:

(1) Let $X$ be a continuum and let $\mathfrak{Q}$ be the hyperspace $2^{X}$ or $C(X)$ of $X$ with the Hausdorff metric. Then if $\omega$ is any Whitney map for $\mathfrak{D}$, for any $0 \leqslant s \leqslant t \leqslant \omega(X)$ $\omega^{-1}(t)$ is an approximate strong deformation retract of $\omega^{-1}([s, t])$. In particular, $\operatorname{Sh}\left(\omega^{-1}(t)\right)=\operatorname{Sh}\left(\omega^{-1}([s, t])\right)$.

(2) Pointed 1-movability is a Whitney property.

(3) For any given $n<\infty$, the property of (cohomological) dimension $\leqslant n$ is a sequential strong Whitney-reversible property.

(4) The property of being chainable or circle-like is a sequential strong Whitneyreversible property.

(5) The property of being an FAR is a Whitney property for 1-dimensional continua.

Property (2) is an affirmative answer to a problem of J. T. Rogers [16, 112]. Properties (3) and (4) are affirmative answers to problems of S. B. Nadler [20, (14.57) and 21].
\end{abstract}

0. Introduction. By a continuum we mean a compact connected metric space. For a given continuum $X$, by the hyperspaces of $X$ we mean

$$
\begin{gathered}
2^{X}=\{A \subset X \mid A \text { is a nonempty closed subset of } X\}, \\
C(X)=\left\{A \in 2^{X} \mid A \text { is connected }\right\},
\end{gathered}
$$

which have the Hausdorff metric $\rho_{\mathfrak{Q}}$ (see [20]). The term mapping is a continuous function. Let $\mathfrak{Q}=2^{X}$ or $C(X)$. A mapping $\omega: \mathfrak{G} \rightarrow[0, \omega(X)]$ is called a Whitney map for $\mathfrak{S}$ [20 or 31] provided that the following conditions are satisfied;

(a) if $A \subset B$ and $A \neq B$, then $\omega(A)<\omega(B)$,

(b) $\omega(\{x\})=0$ for each $x \in X$.

In 1932, H. Whitney [31] showed that Whitney maps always exist. The notion of Whitney map is a convenient and important tool for hyperspace theory. A topological property $\mathrm{P}$ is said to be a Whitney property [20 or $\mathbf{2 1}$ ] provided that whenever a continuum $X$ has property $\mathrm{P}$, so does $\omega^{-1}(t)$ for each Whitney map $\omega$ for $C(X)$ and $0 \leqslant t<\omega(X)$. It is well known that connectibility is a Whitney property. If $\omega$ is a

Received by the editors April 9, 1985 and, in revised form, October 16, 1985.

1980 Mathematics Subject Classification. Primary 54B20; Secondary 54C55, 54C56, 54F45.

Key words and phrases. Hyperspaces, Whitney maps, Whitney property, (sequential) strong Whitneyreversible property, approximate strong deformation retract, pointed 1-movable, FAR, ANR, chainable, circle-like, $\varepsilon$-mapping, indecomposable. 
Whitney map for $\tilde{\mathfrak{p}}=2^{X}$ or $C(X)$, then Whitney levels $\omega^{-1}(t)$ are coverings of $X$ which, as $t$ gets close to zero, converge to $\omega^{-1}(0) \cong X$. Hence, it is of interest to obtain information about the structure of Whitney levels and determine those properties which are preserved by the convergence of positive Whitney levels to the zero level. A topological property $\mathrm{P}$ is said to be a strong Whitney-reversible property [20 or 21] provided that whenever $X$ is a continuum such that $\omega^{-1}(t)$ has property $\mathrm{P}$ for some Whitney map $\omega$ for $C(X)$ and for $0<t<\omega(X)$, then $X$ has property $\mathrm{P}$. The notions of Whitney property and strong Whitney-reversible property are "converses" to each other. A topological property $\mathrm{P}$ is said to be a sequential strong Whitney-reversible property [20 or 21] provided that if $X$ is a continuum such that there is a Whitney map $\omega$ for $C(X)$ and a decreasing sequence $\left\{t_{n}\right\}_{n=1,2, \ldots} \rightarrow 0$ as $n \rightarrow \infty$ such that $\omega^{-1}\left(t_{n}\right)$ has property $\mathrm{P}$ for each $n$, then $X$ has property P. Clearly, sequential strong Whitney-reversible property implies strong Whitney-reversible property.

The purpose of this paper is to investigate Whitney properties, strong Whitneyreversible properties and sequential strong Whitney-reversible properties.

In [20], S. B. Nadler proved that the property of being arcwise connected is a Whitney property. In relation to this result, J. T. Rogers had the following problem $[16,112]$ : Is the property of being pointed 1 -movable a Whitney property? In the first section, we show that for any continuum $X$ and any Whitney map $\omega$ for $2^{X}$ or $C(X), \omega^{-1}(t)$ is an approximate strong deformation retract of $\omega^{-1}([s, t])$ for $0 \leqslant s \leqslant$ $t \leqslant \omega(X)$. As a corollary, it is shown that Rogers' problem has an affirmative answer. Also, it is shown that some shape properties are sequential strong Whitneyreversible properties, e.g., the property of being approximately $n$-connected $\left(=\mathrm{AC}^{n}\right)$ or fundamental dimension $\leqslant n$, etc. In [21], S. B. Nadler proved that for any given $n<\infty$, the property of dimension $\leqslant n$ is a strong Whitney-reversible property. He had the following question [21, (2.10)]: Is the property of dimension $\leqslant n$ a sequential strong Whitney-reversible property? In the second section, we show that for a given ANR $M$, the property of being extendable with respect to $M$ is a sequential strong Whitney-reversible property. As a corollary, we obtain that the property of (cohomological) dimension $\leqslant n$ is a sequential strong Whitney-reversible property. In [10], J. Krasinkiewicz showed that the property of being chainable or proper circle-like is a Whitney property. In [20, (14.57)], S. B. Nadler asked if the converses to Krasinkiewicz' results are true, i.e., is the property of being chainable or circle-like a (sequential) strong Whitney-reversible property? In [21, (5.5), (5.7) and 33], it was shown that the answer to this question is "yes" in the hereditarily indecomposable case and in the hereditarily decomposable case. In $\S 3$, we show that the property of being chainable or circle-like is a sequential strong Whitney-reversible property. In [22], A. Petrus showed that the property of being an FAR is not a Whitney property. More precisely, she showed that there exist a 2-dimensional FAR $X$ (in fact, $X=\mathrm{a}$ disk) and a Whitney map $\omega$ for $C(X)$ such that $\omega^{-1}(t)$ is not an FAR for some $t \in(0, \omega(X))$. Also, she proved that if $X$ is a dendrite (= 1-dimensional AR), then for any Whitney map $\omega$ for $C(X), \omega^{-1}(t)$ is contractible for each $t \in[0, \omega(X)]$. She had the following question: Is the property of being contractible a Whitney property for 1-dimensional continua? In $\S 4$, we prove an "approximation theorem for 
Whitney maps." By using this theorem, we show that the property of being an FAR is a Whitney property for 1-dimensional continua. In the last section, we give some problems that are related to this paper.

Almost all of the results in this paper are proved by the ideas and techniques of ANR and shape theories. The author believes that those theories are also very useful when studying the structures of Whitney maps.

We refer readers to $[\mathbf{1}, \mathbf{4}, \mathbf{8}$ and $\mathbf{1 8}]$ for shape theory and strong shape theory, and to [20] for hyperspaces theory.

1. Shapes of $\omega^{-1}(t)$ and $\omega^{-1}([s, t])$. In this section, we show that for any Whitney map $\omega: \mathfrak{S} \rightarrow[0, \omega(X)]$ for $\mathfrak{S}=2^{X}$ or $C(X), \omega^{-1}(t)$ is an approximate strong deformation retract of $\omega^{-1}([s, t])$ for $0 \leqslant s \leqslant t \leqslant \omega(X)$. In particular, $\operatorname{Sh}\left(\omega^{-1}(t)\right)=$ $\operatorname{Sh}\left(\omega^{-1}([s, t])\right.$. As a corollary, it is shown that pointed 1-movability is a Whitney property. Also, it is shown that some shape properties are sequential strong Whitney-reversible properties.

A subcompactum $X$ of a compactum $Y$ is said to be an approximate strong deformation retract of $Y$ provided that for any ANR $M$ containing $Y$, there exist mappings $r: Y \times[0, \infty) \rightarrow M$ and $D: Y \times[0, \infty) \times I \rightarrow M$ satisfying the conditions

(*) $r(x, t)=x$ for $x \in X$ and $t \in[0, \infty)$, and for any neighborhood $U$ of $X$ in $M$ there is a real $t_{0} \geqslant 0$ such that $r\left(Y \times\left[t_{0}, \infty\right)\right) \subset U$,

(**) $D(x, t, s)=x$ for $x \in X, t \in[0, \infty)$, and $s \in I, D(y, t, 0)=y, D(y, t, 1)=$ $r(y, t)$ for $y \in Y$ and $t \in[0, \infty)$, and for any neighborhood $V$ of $Y$ in $M$ there is a real $t_{1} \geqslant 0$ such that $D\left(Y \times\left[t_{1}, \infty\right) \times I\right) \subset V$.

Clearly, the inclusion $i: X \hookrightarrow Y$ is a (strong) shape equivalence (e.g., see $[1,4,8$ or 18]). Hence $\operatorname{Sh}(X)=\operatorname{Sh}(Y)$. A continuum $X$ is said to be pointed 1-movable [13 or 18] provided that for any ANR $M$ containing $X$ and each neighborhood $U$ of $X$ in $M$, there is a neighborhood $V \subset U$ of $X$ in $M$ such that each loop in $\left(V, x_{0}\right)$ can be deformed within $\left(U, x_{0}\right)$ into any neighborhood of $X$ in $M$. It is well known that planar continua are pointed 1-movable but nonplanar circle-like continua are not pointed 1-movable. Let $X$ be a continuum and $x, y \in X$. Then we say that $x$ and $y$ are joinable in $X[\mathbf{1 3}$ or 18] provided that for any ANR $M$ containing $X$, there is a mapping $\varphi: I \times[0, \infty) \rightarrow M$ satisfying the condition

$\varphi(0, t)=x, \varphi(1, t)=y$ for each $t \in[0, \infty)$, and for each neighborhood $U$ of $X$ in $M$ there is a real $t_{0} \geqslant 0$ such that $\varphi\left(I \times\left[t_{0} \infty\right)\right) \subset U$.

Such a map $\varphi$ is called an approximate path from $x$ to $y$ in $X$. If each pair of points of $X$ is joinable in $X$, then $X$ is said to be joinable.

Note that the properties of being "approximate strong deformation retract" and being "joinable" are not dependent on the choice of ANR $M$ and embeddings of $X$ and $Y$ into $M$ (cf. [1 and 13]).

In relation to pointed 1-movability and joinability, J. Krasinkiewicz and P. Minc proved

(1.1) (J. Krasinkiewicz and P. Minc [13]). A continuum $X$ is pointed 1-movable if and only if $X$ is joinable. In particular, arcwise connected continua (more generally, $\lambda$-connected continua) are pointed 1-movable. 
The following result will be used many times in this paper.

(1.2) (L. E. Ward, Jr. [30]). If $Y$ is a continuum and $X$ is a subcontinuum of $Y$, then any Whitney map for $C(X)\left(\right.$ resp. $\left.2^{X}\right)$ can be extended to a Whitney map for $C(Y)$ (resp. $\left.2^{Y}\right)$.

The main result in this section is the following

(1.3) TheOREM. Let $X$ be a continuum and let $\omega: \mathfrak{g} \rightarrow[0, \omega(X)]$ be any Whitney map for $\mathfrak{B}=2^{X}$ or $C(X)$. Then $\omega^{-1}(t)$ is an approximate strong deformation retract of $\omega^{-1}([s, t])$ for $0 \leqslant s \leqslant t \leqslant \omega(X)$. In particular, $\operatorname{Sh}\left(\omega^{-1}(t)\right)=\operatorname{Sh}\left(\omega^{-1}([s, t])\right)$.

(1.4) Theorem. Let $X$ be a continuum and let $\omega: C(X) \rightarrow[0, \omega(X)]$ be any Whitney map for $C(X)$. Suppose that $A_{1}, A_{2} \in \omega^{-1}\left(\left[0, t_{0}\right]\right)$ for some $t_{0} \in[0, \omega(X)]$ and $A_{1}$ and $A_{2}$ are joinable in $\omega^{-1}\left(\left[0, t_{0}\right]\right)$. If $B_{1}, B_{2} \in \omega^{-1}\left(t_{1}\right)$ for $t_{1} \geqslant t_{0}$ and $A_{i} \cap B_{i} \neq \varnothing(i=1,2)$, then $B_{1}$ and $B_{2}$ are joinable in $\omega^{-1}\left(t_{1}\right)$.

As a corollary of (1.1) and (1.4), we have

(1.5) Corollary. For any Whitney map $\omega$ for $C(X)$, if $\omega^{-1}(t)$ is pointed 1-movable for some $t \in[0, \omega(X)]$, then $\omega^{-1}\left(t^{\prime}\right)$ is also pointed 1 -movable for $t^{\prime} \geqslant t$. In particular, the property of being pointed 1-movable is a Whitney property.

Proof of (1.3). For simplicity, we assume that $s=0$. The case $s>0$ will be proved by the same method. Suppose that $X$ is lying in the Hilbert cube $Q=$ $\prod_{i=0}^{\infty}[-1,1]$. Choose a decreasing sequence $X_{1} \supset X_{2} \supset X_{3} \supset \cdots$ of Peano continua such that for each $n, X_{n}$ is a closed neighborhood of $X$ in $Q$ and $X=\bigcap_{n=1}^{\infty} X_{n}$. By (1.2), there is a Whitney map $\mu: \mathfrak{S}^{\prime} \rightarrow[0, \mu(Q)]$ which is an extension of $\omega$, where $\mathfrak{S}^{\prime}=2^{Q}$ if $\mathfrak{Q}=2^{X}$, and $\mathfrak{Q}^{\prime}=C(Q)$ if $\mathfrak{\mathcal { Q }}=C(X)$. By [32], $\mathfrak{\mathscr { Q }}^{\prime}$ is an ANR. Set $\mu_{X_{n}}=\mu \mid \mathfrak{S}_{n}$, where $\mathfrak{E}_{n}=2^{X_{n}}$ if $\mathfrak{Q}=2^{X}$, and $\mathfrak{E}_{n}=C\left(X_{n}\right)$ if $\mathfrak{Q}=C(X)$. Clearly, $\mu_{X_{11}}$ is a Whitney map for $\mathfrak{Q}_{n}$. Note that $\mu^{-1}(t) \supset \mu_{X_{1}}^{-1}(t) \supset \mu_{X_{2}}^{-1}(t) \supset \cdots$ and $\omega^{-1}(t)=\bigcap_{n=1}^{\infty} \mu_{X_{n}}^{-1}(t)$. Now, for each $n=1,2, \ldots$, we shall construct mappings $r_{n}$ : $\omega^{-1}([0, t]) \rightarrow \mu_{X_{n}}^{-1}(t)$ and homotopies $F_{n}: \omega^{-1}([0, t]) \times I \rightarrow \mu_{X_{n}}^{-1}(t)$ such that $r_{n} \mid \omega^{-1}(t)$ $=$ the identity mapping on $\omega^{-1}(t)$ and $F_{n}: r_{n} \simeq r_{n+1}$ rel. $\omega^{-1}(t)$ in $\mu_{X_{n}}^{-1}(t)$. Since $X_{n}$ is a Peano continuum, $X_{n}$ has a convex metric $d_{n}$. As in $[5,(1.2)$ Theorem], define a homotopy $K_{d_{n}}: \mathfrak{S}_{n} \times[0, \infty) \rightarrow \mathfrak{S}_{n}$ by letting

$$
K_{d_{n}}(A, s)=\left\{x \in X_{n} \mid d_{n}(x, a) \leqslant s \text { for some } a \in A\right\} .
$$

For each $n=1,2, \ldots$, define a function $\left.r_{n}: \omega^{-1}[0, \mathrm{t}]\right) \rightarrow \mu_{X_{n}}^{-1}(t)$ by

$$
r_{n}(A)=K_{d_{n}}\left(A, \theta^{n}(A)\right) \text {, where } \mu_{X_{n}}\left(K_{d_{n}}\left(A, \theta^{n}(A)\right)\right)=t .
$$

Then we can easily see that $r_{n}$ is continuous (cf. [5, (3.1) Proposition]).

Next, we shall construct homotopies $F_{n}(n=1,2, \ldots)$. For this purpose, we need to define homotopies $R_{n}: \omega^{-1}([0, t]) \times I \rightarrow \mu_{X_{n}}^{-1}([0, t])(n=1,2, \ldots)$ by

$R_{n}(A, s)=K_{d_{n}}\left(A, \theta_{s}^{n}(A)\right)$, where $\mu_{X_{n}}\left(K_{d_{n}}\left(A, \theta_{s}^{n}(A)\right)\right)=s \cdot t+(1-s) \mu_{X_{n}}(A)$.

By a way similar to [5, (3.1) Proposition], $R_{n}$ is continuous. Note that $R_{n}(A, 1)=$ $r_{n}(A)$ for each $A \in \omega^{-1}([0, t])$. Also, define homotopies $S_{n}: \omega^{-1}([0, t]) \times I \rightarrow \mu_{X_{n}}^{-1}([0, t])$ 
$(n=1,2, \ldots)$ by

$$
S_{n}(A, s)= \begin{cases}R_{n}(A, 1-2 s) & \text { if } 0 \leqslant s \leqslant 1 / 2, \\ R_{n+1}(A, 2 s-1) & \text { if } 1 / 2 \leqslant s \leqslant 1 .\end{cases}
$$

Set $F_{n}=r_{n} \circ S_{n}: \omega^{-1}([0, t]) \times I \rightarrow \mu_{X_{n}}^{-1}(t)$. Then we have

$$
F_{n}(A, 0)=r_{n} R_{n}(A, 1)=r_{n} r_{n}(A)=r_{n}(A) \text {, }
$$

$$
F_{n}(A, 1)=r_{n} R_{n+1}(A, 1)=r_{n} r_{n+1}(A)=r_{n+1}(A) \text { for } A \in \omega^{-1}([0, t]) \text {, }
$$

and

$$
F_{n}(A, s)=A \text { for } A \in \omega^{-1}(t) \text { and } 0 \leqslant s \leqslant 1 .
$$

\begin{tabular}{|c|c|c|c|c|c|c|c|}
\hline 0 & & 1 & & 2 & & 3 & \\
\hline$\omega^{-1}([0, t])$ & $\gamma_{1} \quad F_{1}$ & $\gamma_{2}$ & $F_{2}$ & $\gamma_{3}$ & $F_{3}$ & $\gamma_{4}$ & $F_{4}$ \\
\hline
\end{tabular}

Hence $F_{n}(n=1,2, \ldots)$ are desired homotopies. By using $F_{n}$, we can easily define a mapping $r: \omega^{-1}([0, t]) \times[0, \infty) \rightarrow \mathfrak{Q}^{\prime}$ satisfying condition $(*)$ (see Figure 1$)$.

FIGURE 1

Next, we shall construct a mapping $D: \omega^{-1}([0, t]) \times[0, \infty) \times I \rightarrow \mathfrak{S}^{\prime}$ satisfying condition (**). For $n=1,2, \ldots$, define mappings $T_{n}: \omega^{-1}\left([0, t] \times I \times I \rightarrow \mu_{X_{n}}^{-1}([0, t])\right.$ by

$$
T_{n}\left(A, s, s^{\prime}\right)=K_{d_{n}}\left(S_{n}(A, s), \theta_{s^{\prime}}^{n}\left(S_{n}(A, s)\right)\right),
$$

where

$$
\mu_{X_{n}}\left(K_{d_{n}}\left(S_{n}(A, s), \theta_{s^{\prime}}^{n}\left(S_{n}(A, s)\right)\right)\right)=s^{\prime} \cdot t+\left(1-s^{\prime}\right) \mu_{X_{n}}\left(S_{n}(A, s)\right) .
$$

By using the homotopies $T_{n}$, we can easily obtain homotopies $D_{n}: \omega^{-1}([0, t]) \times I \times I$ $\rightarrow \mu_{X_{u}}^{-1}([0, t])$ such that

$$
\begin{gathered}
D_{n}(A, s, 0)=A, \quad D_{n}(A, s, 1)=F_{n}(A, s), \\
D_{n}\left(A, 0, s^{\prime}\right)=R_{n}\left(A, s^{\prime}\right), \quad D_{n}\left(A, 1, s^{\prime}\right)=R_{n+1}\left(A, s^{\prime}\right)
\end{gathered}
$$


for $A \in \omega^{-1}([0, t])$, and

$$
D_{n}\left(A, s, s^{\prime}\right)=A \quad \text { for } A \in \omega^{-1}(t) \text { and } 0 \leqslant s, s^{\prime} \leqslant 1 .
$$

By using $D_{n}$, we can define a mapping $D: \omega^{-1}([0, t]) \times[0, \infty) \times I \rightarrow \mathfrak{S}^{\prime}$ satisfying condition $(* *)$ (see Figure 2).

To prove (1.4), we need the following lemma.

(1.6) Lemma. Let $X$ be a subcontinuum of a continuum $Y$ and let $x, y \in X$. If $X$ is an approximate strong deformation retract of $Y$, and $x$ and $y$ are joinable in $Y$, then $x$ and $y$ are also joinable in $X$.

Proof. Assume that $M$ is an ANR which contains $Y$. Let $\gamma: Y \times[0, \infty) \rightarrow M$ be a mapping satisfying condition (*). Since $x$ and $y$ are joinable in $Y$, there is an approximate path $\varphi: I \times[0, \infty) \rightarrow M$ from $x$ to $y$ in $Y$. Since $M$ is an ANR, there is a neighborhood $W$ of $Y \times[0, \infty)$ in $M \times[0, \infty)$ and an extension $\tilde{\gamma}: W \rightarrow M$ of $\gamma$. By changing the parameter $t \in[0, \infty)$, we may assume that $(\varphi(I \times\{t\}), t) \subset W$ for each $t \in[0, \infty)$. Define a mapping $\varphi^{\prime}: I \times[0, \infty) \rightarrow M$ by $\varphi^{\prime}(s, t)=\tilde{\gamma}(\varphi(s, t), t)$ for $(s, t) \in I \times[0, \infty)$. Clearly, $\varphi^{\prime}$ is an approximate path from $x$ to $y$ in $X$.

Proof OF (1.4). By [7], there are segments $\alpha_{i}: I \rightarrow \omega^{-1}\left(\left[0, t_{1}\right]\right)$ such that $\alpha_{i}(0)=A_{i}$ and $\alpha_{i}(1) \in \omega^{-1}\left(t_{1}\right)$ for $i=1,2$. Then $\alpha_{i}(1) \cap B_{i} \neq \varnothing \quad(i=1,2)$, hence by [20, (14.8.1)] there exist $\operatorname{arcs} \beta_{i}$ in $\omega^{-1}\left(t_{1}\right)$ from $\alpha_{i}(1)$ to $B_{i}(i=1,2)$. Since $A_{1}$ and $A_{2}$ are joinable in $\omega^{-1}\left(\left[0, t_{0}\right]\right), B_{1}$ and $B_{2}$ are joinable in $\omega^{-1}\left(\left[0, t_{1}\right]\right)$. By (1.3) and (1.6), $B_{1}$ and $B_{2}$ are joinable in $\omega^{-1}\left(t_{1}\right)$. This completes the proof.

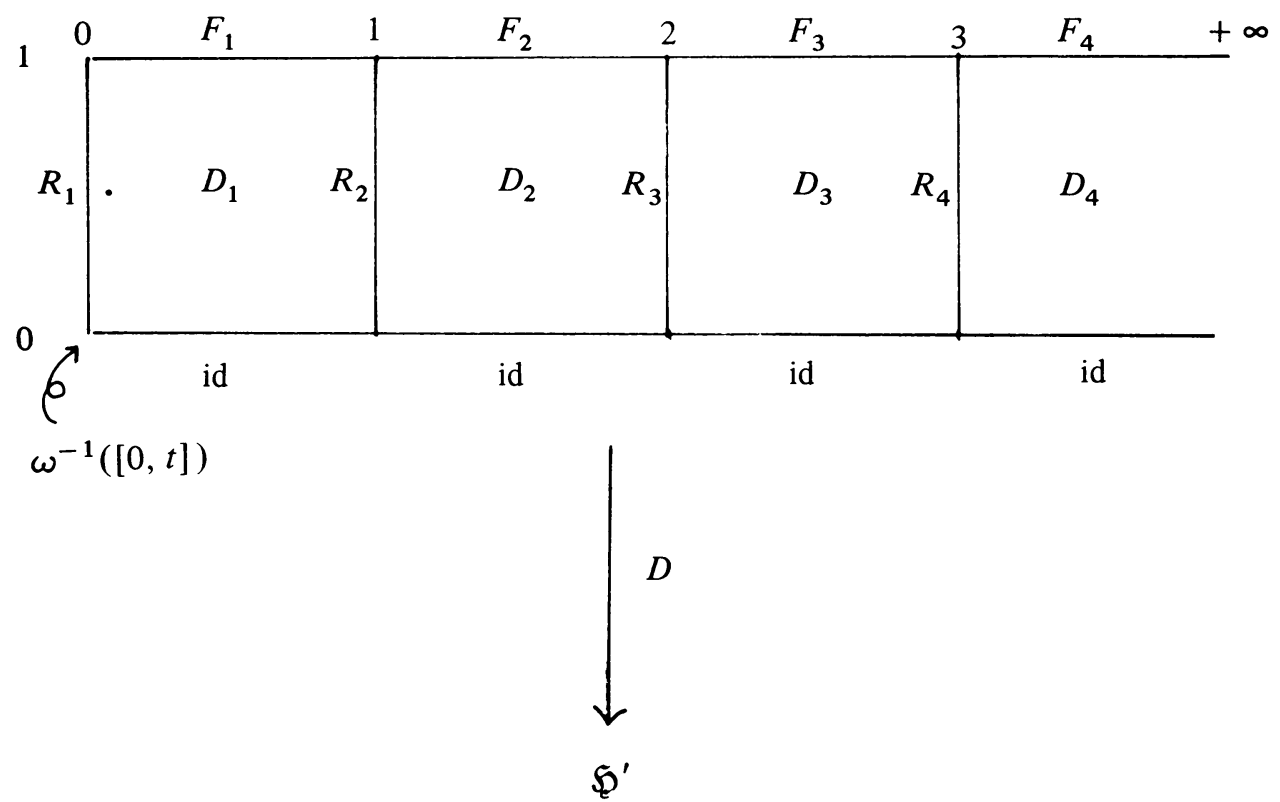

FIGURE 2 
(1.7) RemarK. In (1.4) and (1.5), we cannot replace "Whitney map for $C(X)$ " by "Whitney map for $2^{X}$." In fact, it is well known that there is a Whitney map $\omega$ : $2^{I} \rightarrow[0, \omega(I)]$ such that for some $t \in(0, \omega(I)), \omega^{-1}(t)$ is not connected (see [20, (14.61)]).

(1.8) Example. It is easily seen that if $X$ is a Peano continuum, then for any Whitney map $\omega$ for $\mathfrak{G}=2^{X}$ or $C(X), \omega^{-1}(t)$ is a strong deformation retract of $\omega^{-1}([s, t])$ for $0 \leqslant s \leqslant t \leqslant \omega(X)$. For such a case, this is a stronger result than (1.3): But, if $X$ is not a Peano continuum, $\omega^{-1}(t)$ is not always a strong deformation retract of $\omega^{-1}([s, t])$. Moreover, $\omega^{-1}(t)$ is not always homotopy equivalent to $\omega^{-1}([s, t])$. Consider the following subsets $X, Y$ in the plane $E^{2}$ and $Z$ in the Euclidean 3-dimensional space $E^{3}$ (see Figure 3).

$$
\begin{aligned}
X= & \operatorname{Cl}\left\{(x, \sin [1 / x]) \in E^{2} \mid 0<x \leqslant 1\right\} \\
& \cup \operatorname{Cl}\left\{(x, 2+\sin [1 / x]) \in E^{2} \mid-1 \leqslant x<0\right\}, \\
Y= & C l\left\{(X, \sin [1 / x]) \in E^{2} \mid 0<x \leqslant 1\right\} \cup\left\{(0, y) \in E^{2} \mid 1 \leqslant y \leqslant 2\right\} \\
& \cup C l\left\{(x, 3+\sin [1 / x]) \in E^{2} \mid-1 \leqslant x<0\right\}, \\
Z= & \text { the mapping cylinder of the mapping } f: Y \rightarrow X \text { defined by } f \mid A \\
= & \left\{(0, y) \in E^{2} \mid 1 \leqslant y \leqslant 2\right\} \\
= & \text { the constant map to the point }(0,1) \in E^{2} \text { and } f \mid Y-A \text { is one-to-one. }
\end{aligned}
$$

Let $\omega$ be any Whitney map for $C(X)$. Then there is $t_{0} \in(0, \omega(X))$ such that if $0<t \leqslant t_{0}$, then $\omega^{-1}(t)$ is homeomorphic to $Y$ (see [20, (14.43.2)]). Also, $\omega^{-1}([0, t])$ is homeomorphic to $Z$. Since $\omega^{-1}([0, t])$ is homotopy equivalent to $\omega^{-1}(0) \cong X$ and $X$ and $Y$ are not homotopy equivalent, $\omega^{-1}(t) \cong Y$ is not homotopy equivalent to $\omega^{-1}([0, t])$. Note that for some $\varepsilon>0$ and any $t \in(0, \omega(X))$ there is no mapping $g$ : $\omega^{-1}(0) \rightarrow \omega^{-1}(t)$ such that $\rho_{\mathfrak{p}}(\{x\}, g(\{x\}))<\varepsilon$ for $x \in X$ (cf. (3.1)).

In (1.3), if $s=0$ and $t=\omega(X)$, we have the following well-known result.

(1.9) (J. KRASINKIEWICZ [12]). If $X$ is a continuum, then $\operatorname{Sh}(C(X))=\operatorname{Sh}\left(2^{X}\right)=*$.

(1.10) Corollary. Let $X$ be a continuum and let $\omega$ be a Whitney map for $\mathfrak{G}=2^{X}$ or $C(X)$. Suppose that $t_{1}>t_{2}>t_{3}>\cdots$ is a decreasing sequence of positive numbers in $[0, \omega(X)]$ and $t=\lim _{n \rightarrow \infty} t_{n}$. Then

(1) (S. B. NADLER [21] OR A. KoyAmA [9]). If $\omega^{-1}\left(t_{n}\right)$ is contractible with respect to an ANR $M$, then $\omega^{-1}$ is contractible with respect to $M$. In particular, the property of being an FAR is a sequential strong Whitney-reversible property.

(2) (S. B. NADLER [21]). If $\check{H}^{i}\left(\omega^{-1}\left(t_{n}\right)\right)=0$, then $\check{H}^{i}\left(\omega^{-1}(t)\right)=0$.

(3) If $\omega^{-1}\left(t_{n}\right)$ is approximately m-connected $\left(=\mathrm{AC}^{m}\right)$, then $\omega^{-1}(t)$ is $\mathrm{AC}^{m}$.

(4) If pro- $H_{i}\left(\omega^{-1}\left(t_{n}\right)\right)=0$, then pro- $H_{i}\left(\omega^{-1}(t)\right)=0$. Also, if $\breve{H}_{i}\left(\omega^{-1}\left(t_{n}\right)\right)=0$, then $\check{H}_{i}\left(\omega^{-1}(t)\right)=0$.

(5) If $\operatorname{Fd}\left(\omega^{-1}\left(t_{n}\right)\right) \leqslant m$, then $\operatorname{Fd}\left(\omega^{-1}(t)\right) \leqslant m$.

(See [1 and 18] for the definitions in statements (3), (4), and (5).) 

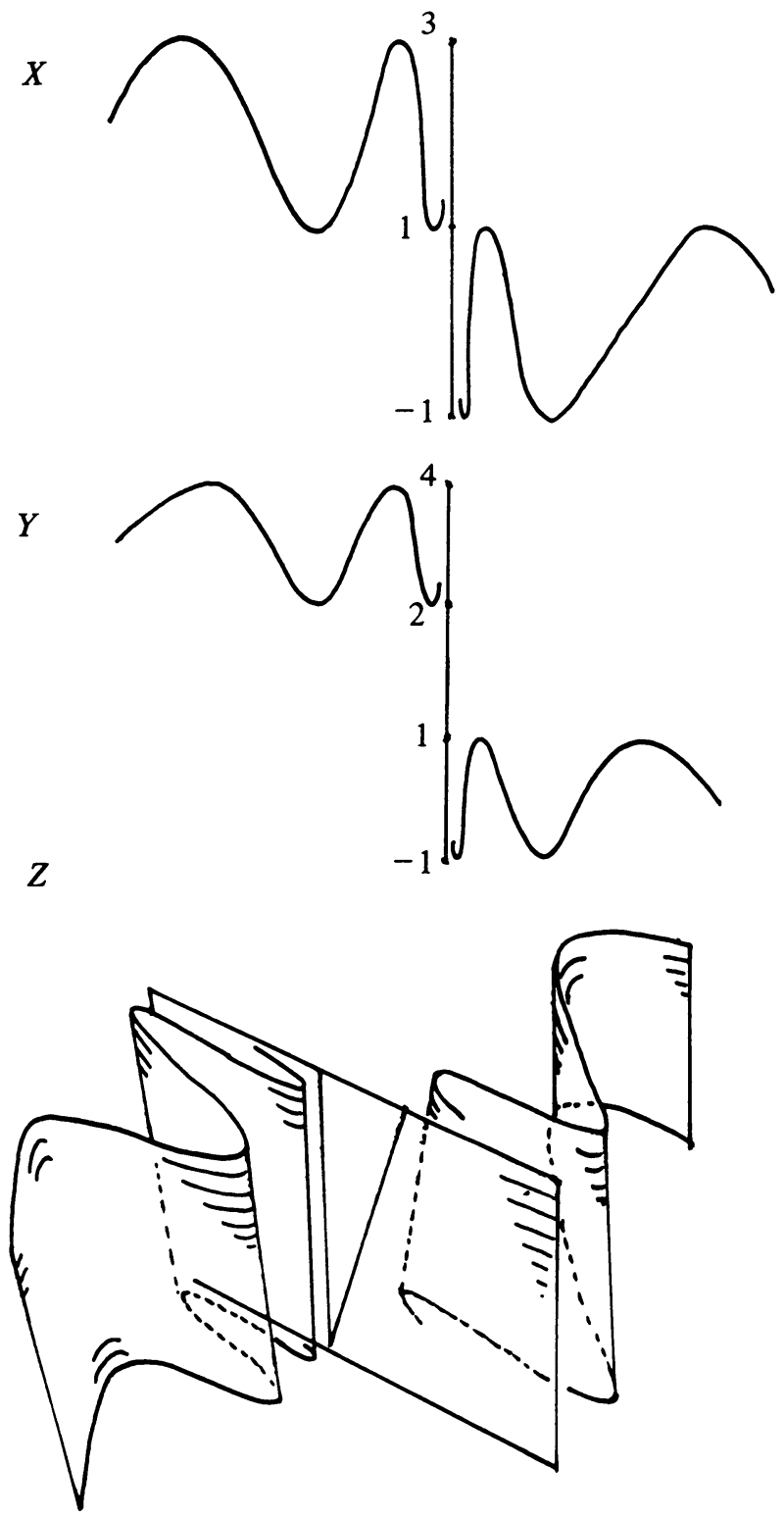

FIGURE 3

Proof. Note that $\omega^{-1}(t)=\bigcap_{n=1}^{\infty} \omega^{-1}\left(\left[t, t_{n}\right]\right)$. The proof follows from the following commutative diagram in shape category (see $[1,18]$ for the definition of shape category):

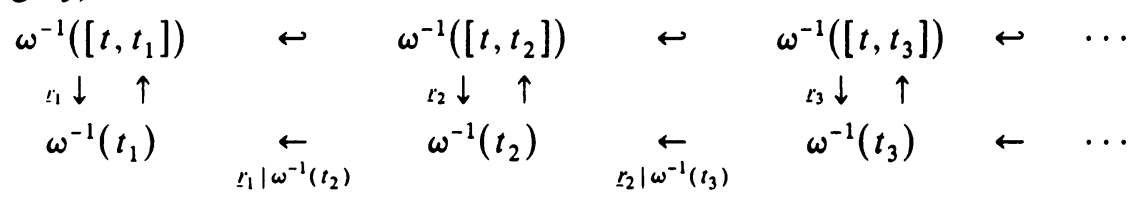


where $\underline{r}_{n}: \omega^{-1}\left(\left[t, t_{n}\right]\right) \rightarrow \omega^{-1}\left(t_{n}\right)$ is a (strong) shape equivalence that is induced by the maping $r_{n}: \omega^{-1}\left(\left[t, t_{n}\right]\right) \times[0, \infty) \rightarrow \mathfrak{S}^{\prime}$ as in the proof of (1.3). By continuity of shape, (1)-(5) are easily proved.

(1.11) CoRollaRY. Let $X$ be a continuum and let $\omega$ be any Whitney map for $\mathfrak{S}=2^{X}$ or $C(X)$. If $X$ is an FANR, there is $t_{0} \in(0, \omega(X))$ such that $\operatorname{Sh}(X) \leqslant \operatorname{Sh}\left(\omega^{-1}(t)\right)$ for each $t \in\left[0, t_{0}\right]$.

Proof. Since $X$ is an FANR, by [3], there is $t_{0} \in(0, \omega(X))$ such that $\omega(0)$ is an approximate retract of $\omega^{-1}\left(\left[0, t_{0}\right]\right)$, i.e., there is a mapping $r: \omega^{-1}\left(\left[0, t_{0}\right]\right) \times[0, \infty) \rightarrow$ $M$ satisfying condition $(*)$, where $M$ is an ANR that contains $\omega^{-1}(0)$. Hence $\omega^{-1}(0)$ is (strong) shape dominated by $\omega^{-1}\left(\left[0, t_{0}\right]\right)$. Since $\omega^{-1}([0, t])$ is (strong) shape equivalent to $\omega^{-1}(t), X$ is (strong) shape domainated by $\omega^{-1}(t)$ for $0 \leqslant t \leqslant t_{0}$.

(1.12) EXAMPLE. In the statement of (1.11), we cannot conclude that $\operatorname{Sh}(X)=$ $\operatorname{Sh}\left(\omega^{-1}(t)\right)$ for $0 \leqslant t \leqslant t_{0}$. Consider the following sets (see [22, Example 5]. In the Euclidean 3-dimensional space $E^{3}$, let $A_{0}=\left\{(x, y, z) \in E^{3} \mid x^{2}+y^{2}=1, z=0\right\}$ and $A_{n}=\left\{(x, y, z) \in E^{3} \mid x^{2}+y^{2}=1, z=1 / 2^{n-1}\right\}(n=1,2, \ldots)$. Let $X_{n}$ be the annulus that is contained in the sphere with center $\left(0,0,3 / 2^{n+1}\right)$ and radius $\sqrt{1+\left(1 / 2^{n+1}\right)}$ and is bounded by circles $A_{n}$ and $A_{n+1}$. Let $\overline{Y=\cup_{n=1}^{\infty} X_{n}}, Z=$ $\left\{(x, y, z) \in E^{3} \mid x^{2}+y^{2} \leqslant 1\right.$ and $\left.z=1\right\}$, and $T=Y \cup Z$. Let $P_{n}$ be paths in $E^{3}$ and let $T_{n}$ be a reduced copy of $T$ in $E^{3}$ such that $\lim \operatorname{diam} T_{n}=0, \lim \operatorname{diam} P_{n}=0$, $P_{i} \cap P_{j}=\varnothing, T_{i} \cap T_{j}=\varnothing(i \neq j)$, and $P_{i} \cap T_{i}=\{*\}, P_{i} \cap T_{i+1}=\{*\}$ as below (see Figure 4). Set $X=\bar{\cup}_{n=1}^{\infty} P_{n} \cup \cup_{n=1}^{\infty} T_{n}$. Note that $X$ is an AR, hence an FAR. Let $S_{n}=\left\{(x, y, z) \in E^{3} \mid\left(x-\left(3 / 2^{n+1}\right)\right)^{2}+y^{2}=\left(1 / 2^{n+1}\right)^{2}\right\} \quad(n=1,2, \ldots)$ and let $S=\overline{\cup_{n-1}^{\infty} S_{n}}$ (see Figure 4). Let $\omega: C(X) \rightarrow[0, \omega(X)]$ be the Whitney map for $C(X)$ as defined by [31] and the usual Euclidean metric. Then, by similar argument as in [22, Example 5], there is a decreasing sequence $t_{1}>t_{2}>\cdots$ of positive numbers in $[0, \omega(X)]$ such that $\lim t_{n}=0$ and for each $n$ there exist mappings $f_{n}$ : $\omega^{-1}\left(t_{n}\right) \rightarrow S$ and $g_{n}: S \rightarrow \omega^{-1}\left(t_{n}\right)$ such that $f_{n} g_{n}=1_{s}$. Since $S$ is not an FANR, $\omega^{-1}\left(t_{n}\right)$ is not an FANR, i.e., the property of being FANR is not a Whitney property. Also, $\operatorname{Sh}\left(\omega^{-1}\left(t_{n}\right)\right) \neq \operatorname{Sh}(X)=*$.

In [29], J. T. Rogers showed that there is always a monomorphism $\check{H}^{1}\left(\omega^{-1}(t)\right) \rightarrow$ $\check{H}^{1}(X)$ for any continuum $X$, any Whitney map $\omega$ for $C(X)$, and $0 \leqslant t \leqslant \omega(X)$. In [15], A. Y. Lau showed that if $X$ is a 1-dimensional continuum and $\check{H}^{1}(X)$ is finitely generated, then there is $0<t \leqslant \omega(X)$ such that $\check{H}^{1}(X) \cong H^{1}\left(\omega^{-1}(s)\right)$ for each $s \leqslant t$. Now, we shall give more precise information about the morphism $\check{H}^{1}\left(\omega^{-1}(t)\right)$ $\rightarrow \check{H}^{1}(X)$ and show that in the result of Lau the condition that $X$ is 1-dimensional is not essential.

Let $r: \omega^{-1}([s, t]) \times[0, \infty) \rightarrow \mathscr{S}^{\prime}$ be the approximate retraction from $\omega^{-1}([s, t])$ to $\omega^{-1}(t)$ as in the proof of (1.3). Let $f_{s t}: \omega^{-1}(s) \rightarrow \omega^{-1}(t)$ be the shape morphism induced by the restriction $r \mid \omega^{-1}(s) \times[0, \infty)$. Note that $f_{t u} f_{s t}=f_{s u}$ for $s \leqslant t \leqslant u$. Then we have the following

(1.13) Corollary (CF. J. T. Rogers [29] AND A. Y. LaU [15]). Let $X$ be $a$ continuum and let $\omega$ be any Whitney map for $C(X)$. Then $\check{H}\left(f_{s t}\right): \check{H}^{1}\left(\omega^{-1}(t)\right) \rightarrow$ $\check{H}\left(\omega^{-1}(s)\right)$ is a monomorphism for $0 \leqslant s \leqslant t \leqslant \omega(X)$. 

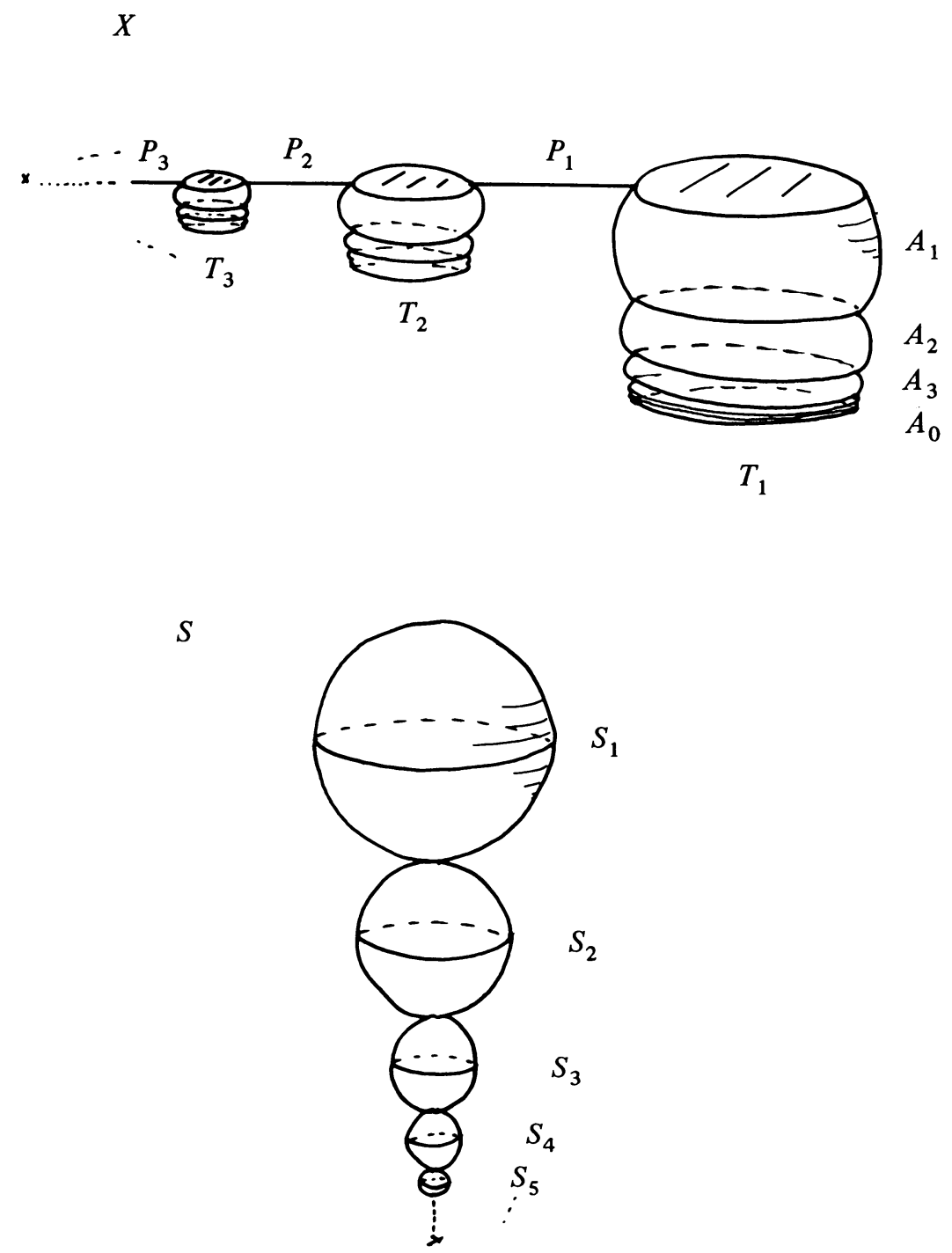

FIGURE 4

Proof. Consider the following exact sequence of Čech cohomology:

$$
\check{H}^{1}\left(\omega^{-1}([0, t]), \omega^{-1}(0)\right) \rightarrow \check{H}^{1}\left(\omega^{-1}([0, t])\right) \stackrel{\check{H}^{1}(i)}{\rightarrow} \check{H}^{1}\left(\omega^{-1}(0)\right),
$$

where $i$ : $\omega^{-1}(0) \hookrightarrow \omega^{-1}([0, t])$ is the inclusion mapping. By [15, Theorem 2], $\check{H}^{1}\left(\omega^{-1}([0, t]), \omega^{-1}(0)\right)=0$. Hence $\check{H}^{1}(i)$ is a monomorphism. By using the homotopy $D$ (see the proof of (1.3)), we can easily see that $j f_{0 t}=i$, where $j: \omega^{-1}(t) \hookrightarrow$ $\omega^{-1}([0, t])$ is the inclusion mapping. Since $j$ is a shape equivalence, $\check{H}^{1}\left(f_{0 t}\right)$ is a monomorphism. Let $0 \leqslant s \leqslant t$. Since $f_{0 t}=f_{s t} f_{0 s}, \breve{H}^{1}\left(f_{s t}\right)$ is a monomorphism. 
(1.14) Corollary. Let $X$ be a continuum and let $\omega$ be any Whitney map for $C(X)$. Suppose that $\check{H}^{1}(X)$ is finitely generated. Then for any $s \in[0, \omega(X))$, there is $s^{\prime}>s$ such that $\breve{H}^{1}\left(f_{s t}\right)$ : $\breve{H}^{1}\left(\omega^{-1}(t)\right) \rightarrow \breve{H}^{1}\left(\omega^{-1}(s)\right)$ is an isomorphism for each $s \leqslant t \leqslant s^{\prime}$.

Proof. Since $\check{H}^{1}\left(f_{0 s}\right): \check{H}^{1}\left(\omega^{-1}(s)\right) \rightarrow \check{H}^{1}(X)$ is a monomorphism, $\check{H}^{1}\left(\omega^{-1}(s)\right)$ is also finitely generated. We may assume that $\breve{H}^{1}\left(\omega^{-1}(s)\right)=\left[\omega^{-1}(s), S^{1}\right]$, where $S^{1}$ is a circle and $[Y, Z]$ denotes the homotopy classes of mappings from $Y$ to $Z$. Let $\left[g_{1}\right]$, $\left[g_{2}\right], \ldots,\left[g_{n}\right]$ be generators of $\check{H}^{1}\left(\omega^{-1}(s)\right)$, where $g_{i}: \omega^{-1}(s) \rightarrow S^{1}$ is a mapping $(i=1,2, \ldots, n)$. Since $S^{1}$ is an ANR, there is $s^{\prime}>s$ and extensions $\tilde{g}_{i}: \omega^{-1}\left(\left[s, s^{\prime}\right]\right)$ $\rightarrow S^{1}(i=1,2, \ldots, n)$ of $g_{i}$. Let $s \leqslant t \leqslant s^{\prime}$. Then $\check{H}^{1}(i)\left(\left[\tilde{g}_{i}\right]\right)=\left[g_{i}\right](i=1,2, \ldots, n)$, where $i: \omega^{-1}(s) \hookrightarrow \omega^{-1}([s, t])$ is the inclusion mapping. Hence $\check{H}^{1}(i)$ is an epimorphism. Note that $i=j f_{s t}$, where $j: \omega^{-1}(t) \hookrightarrow \omega^{-1}([s, t])$ is the inclusion mapping. Hence $\check{H}^{1}(i)=\check{H}^{1}\left(f_{s t}\right) \breve{H}^{1}(j)$, which implies that $\breve{H}^{1}\left(f_{s t}\right)$ is an epimorphism. By (1.13), $\breve{H}^{1}\left(f_{s t}\right)$ is an isomorphism. This completes the proof.

(1.15) EXAMPLE. In the statement of (1.14), we cannot omit the condition that $\check{H}^{1}(X)$ is finitely generated. Consider the set $X=\overline{\bigcup_{n=1}^{\infty} S_{n}}$ in the plane $E^{2}$, where $S_{n}$ is the circle in $E^{2}$ with center $((n-1) / n, 0)$ and radius $1 / n$. Note that $\check{H}^{1}(X)$ is not finitely generated. Now, we shall show that for each $t \in(0, \omega(X)], \breve{H}^{1}\left(f_{0 t}\right)$ : $\check{H}^{1}\left(\omega^{-1}(t)\right) \rightarrow \breve{H}^{1}(X)$ is not an epimorphism. Consider the morphism $\check{H}^{1}(i)$ : $\left[\omega^{-1}([0, t]), S^{1}\right] \rightarrow\left[X, S^{1}\right]$, where $i: X \hookrightarrow \omega^{-1}([0, t])$ is the natural inclusion mapping. Since $\lim _{n \rightarrow \infty} \operatorname{diam} S_{n}=0$, there is $n_{0}$ such that $C\left(S_{n}\right) \subset \omega^{-1}([0, t])$ for $n \geqslant n_{0}$. Let $g_{n}: X \rightarrow S^{1}$ be a mapping such that $g_{n} \mid S_{n}=$ a homeomorphism. Since $C\left(S_{n}\right)$ is a disk, Image $\check{H}^{1}(i) \nexists \nexists\left[g_{n}\right]\left(n \geqslant n_{0}\right)$. Since $i=\check{J j}_{0 t}$ and $j$ is a shape equivalence, Image $\check{H}^{1}\left(f_{0 t}\right) \nexists \nexists\left[g_{n}\right]\left(n \geqslant n_{0}\right)$. Hence $\check{H}^{1}\left(f_{0 t}\right)$ is not an epimorphism.

2. Extendability with respect to an ANR. In [21], it is shown that for any given $n<\infty$, the property of dimension $\leqslant n$ is a strong Whitney-reversible property. Also, S. B. Nadler had the following question [21, (2.10)]: Is the property of dimension $\leqslant n$ is a sequential strong Whitney-reversible property? In this section, we prove that the property of being extendable with respect to an ANR $M$ is a sequential strong Whitney-reversible property. As a corollary, it is shown that Nadler's question has an affirmative answer.

Let $M$ be an ANR. A continuum $X$ is said to be extendable with respect to $M$ provided that for any closed subset $A$ of $X$ and any mapping $f: A \rightarrow M$, there exists an extension $\tilde{f}: X \rightarrow M$ of $f$.

The following is the main theorem in this section.

(2.1) TheOREM. Let $M$ be an ANR and let $X$ be a continuum. Suppose that $\omega$ : $\mathfrak{S} \rightarrow[0, \omega(X)]$ is a Whitney map for $\mathfrak{S}=2^{X}$ or $C(X)$ and $t_{1}>t_{2}>\cdots$ is a decreasing sequence of positive numbers in $[0, \omega(X)]$ such that $\lim t_{n}=t$. If $\omega^{-1}\left(t_{n}\right)$ is extendable with respect to $M$, then $\omega^{-1}(t)$ is extendable with respect to $M$.

If we consider the case $\mathfrak{E}=C(X)$ and $M=n$-sphere $S^{n}$ or $M=$ the EilenbergMac Lane complex $K(Z, n)$, then we have the following

(2.2) Corollary. For any given $n<\infty$, the property of (cohomological) dimension $\leqslant n$ is a sequential strong Whitney-reversible property. 
To prove (2.1), we need the following

(2.3) (J. L. KeLLEY [7, (1.5)]). Let $X$ be a continuum and let $\omega$ be any Whitney map for $\mathfrak{Q}=2^{X}$ or $C(X)$. Then for any $\varepsilon>0$ there is $\eta(\varepsilon)>0$ such that if $A, B \in \mathfrak{Q}$, $A \subset B$, and $\omega(B)-\omega(A)<\eta(\varepsilon)$, then $\rho_{\mathfrak{q}}(A, B)<\varepsilon$, where $\rho_{\mathfrak{\Phi}}$ denotes the Hausdorff metric on $\mathfrak{S}$.

(2.4) THEOREM. Let $X$ be a continuum and let $\omega$ be any Whitney map for $\mathfrak{\mathscr { Q }}=2^{X}$ or $C(X)$. Suppose that $M$ is an ANR that contains $\mathfrak{Q}$. Then, for any $\varepsilon>0$ there is $\eta(\varepsilon)>0$ such that if $0 \leqslant t \leqslant t^{\prime} \leqslant \omega(X)$ and $t^{\prime}-t<\eta(\xi)$, then $\omega^{-1}\left(t^{\prime}\right)$ is an $\varepsilon$-approximate strong deformation retract of $\omega^{-1}\left(\left[t, t^{\prime}\right]\right)$ in $M$.

Let $Y$ be a compactum in an ANR $M$. A subcompactum $X$ of $Y$ is said to be an $\varepsilon$-approximate strong deformation retract of $Y$ in $M$ provided that there exist mappings $r: Y \times[0, \infty) \rightarrow M$ and $D: Y \times[0, \infty) \times I \rightarrow M$ satisfying conditions $(*),(* *)$ and

$(* * *) \quad d(y, D(y, s, t))<\varepsilon$ for any $y \in Y, s \in[0, \infty)$ and $t \in I$.

Note that $Y$ is contained in the $\varepsilon$-neighborhood of $X$ in $M$.

SKETCH OF THE PROOF OF (2.4). Assume that $X$ is lying in the Hilbert cube $Q$ and $\mu: \mathfrak{Q}^{\prime} \rightarrow[0, \mu(Q)]$ is an extension of $\omega$, where $\mathfrak{S}^{\prime}=2^{Q}$ or $C(Q)$. Let $i: U \rightarrow M$ be an extension of $i_{\mathfrak{S}}: \mathfrak{Q} \rightarrow \mathfrak{G}$, where $U$ is a neighborhood of $\mathfrak{\mathscr { E }}$ in $\mathfrak{Q}^{\prime}$. Choose $\varepsilon^{\prime}>0$ such that if $\rho^{\prime}(A, B)<\varepsilon^{\prime}$, then $d(i(A), i(B))<\varepsilon$. By $(2.3)$, there is $\eta\left(\varepsilon^{\prime}\right)>0$ as in (2.3) for $\mu: \mathfrak{S}^{\prime} \rightarrow[0, \mu(Q)]$. We shall show that $\eta\left(\varepsilon^{\prime}\right)$ is a desired positive number. Let $t, t^{\prime} \in[0, \omega(X)], t \leqslant t^{\prime}$, and $t^{\prime}-t<\eta\left(\varepsilon^{\prime}\right)$. In the proof of (1.3), we can see that $r_{n}(A) \supset A, R_{n}(A, s) \supset A, S_{n}(A, s) \supset A$, and $F_{n}(A, s) \supset A$ for each $n=$ $1,2, \ldots, A \in \omega^{-1}\left(\left[t, t^{\prime}\right]\right)$, and $0 \leqslant s \leqslant 1$. Hence we conclude that $D\left(A, s, s^{\prime}\right) \supset A$ for $A \in \omega^{-1}\left(\left[t, t^{\prime}\right]\right), s \in[0, \infty)$, and $s^{\prime} \in I$. Note that $\mu\left(D\left(A, s, s^{\prime}\right)\right)-\mu(A)<\eta\left(\varepsilon^{\prime}\right)$. By (2.3), $\rho_{s^{\prime}}\left(D\left(A, s, s^{\prime}\right), A\right)<\varepsilon^{\prime}$ for $A \in \omega^{-1}\left(\left[t, t^{\prime}\right]\right)$. Without loss of generality, we may assume that $D\left(\omega^{-1}\left(\left[t, t^{\prime}\right]\right) \times[0, \infty) \times I\right) \subset U$. Then $\tilde{D}=i \circ D: \omega^{-1}\left(\left[t, t^{\prime}\right]\right) \times$ $[0, \infty) \times I \rightarrow M$ is a desired mapping.

Proof of (2.1). Let $N$ be an ANR that contains $H$. Let $\Lambda$ be any closed subset of $\omega^{-1}(t)$ and $f: \Lambda \rightarrow M$ be any mapping. Since $M$ is an ANR, there is a closed neighborhood $U$ of $\Lambda$ in $N$ and there is an extension $f_{1}: U \rightarrow M$ of $f$. Take a positive number $\varepsilon$ such that $\{A \in N \mid d(A, B) \leqslant \varepsilon$ for some $B \in \Lambda\} \subset \operatorname{Int} U$. Let $\eta(\varepsilon)$ be the positive number as in (2.4). Choose $t_{n}$ such that $t_{n}-t<\eta(\varepsilon)$. Since $\omega^{-1}\left(t_{n}\right)$ is extendable with respect to $M$, there is an extension $g: \omega^{-1}\left(t_{n}\right) \rightarrow M$ of $f_{1} \mid U \cap \omega^{-1}\left(t_{n}\right)$. Since $M$ is an ANR, there is a neighborhood $W$ of $\omega^{-1}\left(t_{n}\right)$ in $N$ and an extension $\tilde{g}$ : $U \cup W \rightarrow M$ of $g$ and $f_{1}$. By (2.4), there is a mapping $D$ : $\omega^{-1}\left(\left[t, t_{n}\right]\right) \times[0, \infty) \times I \rightarrow N$ satisfying conditions $(*),(* *)$ and $(* * *)$. Choose $s_{0} \in$ $[0, \infty)$ such that $D\left(\omega^{-1}\left(\left[t, t_{n}\right]\right) \times\left[s_{0}, \infty\right) \times\{1\}\right) \subset W$. Consider the homotopy $H$ : $\omega^{-1}(t) \times I \rightarrow N$ defined by $H=D \mid \omega^{-1}(t) \times\left\{s_{0}\right\} \times I$. Then $H(A, 0)=A, H(A, 1)$ $\in W$, and $d(H(A, s), A)<\varepsilon$ for $A \in \omega^{-1}(t)$ and $0 \leqslant s \leqslant 1$. Note that $H(\Lambda \times I)$ $\subset$ Int $U$. Let $V$ be a neighborhood of $\Lambda$ in $\omega^{-1}(t)$ such that $H(V \times I) \subset U$. Choose 
a mapping $\alpha: \omega^{-1}(t) \rightarrow I$ such that $\alpha(A)=0$ if $A \in \Lambda$ and $\alpha(A)=1$ if $A \in \omega^{-1}(t)$ $-V$. Then $H(A, \alpha(A)) \in U \cup W$ for $A \in \omega^{-1}(t)$. Define a mapping $\tilde{f}: \omega^{-1}(t) \rightarrow M$ by

$$
\tilde{f}(A)=\tilde{g} H(A, \alpha(A)) \quad \text { for } A \in \omega^{-1}(t) .
$$

Clearly, $\tilde{f}$ is an extension of $f$. Hence $\omega^{-1}(t)$ is extendable with respect to $M$. This completes the proof.

3. The property of being chainable or circle-like is a sequential strong Whitneyreversible property. In [10], J. Krasinkiewicz proved that the property of being chainable or proper circle-like is a Whitney property. In [20, (14.57)], S. B. Nadler asked if the converses are true, i.e., is the property of being (a) chainable or (b) circle-like a (sequential) strong Whitney-reversible property? In [21, (5.5), (5.7) and 33], it was shown that the answer is "yes" in the hereditarily indecomposable case and hereditarily decomposable case. In this section, we show that the property of being chainable or circle-like is a sequential strong Whitney-reversible property.

In this section, we need the following definitions. A mapping $f: X \rightarrow Y$ between compacta is said to be an $\varepsilon$-mapping provided that for each $y \in Y$, diam $f^{-1}(y)<\varepsilon$ $\left(f^{-1}(y)\right.$ may be empty). In this paper, " $\varepsilon$-mapping" does not imply "surjective." Let $\mathfrak{B}$ be a given ANR-family. A compactum $X$ is said to be weak $\mathfrak{B}$-like provided that for each $\varepsilon>0$ there is an $\varepsilon$-mapping from $X$ to some $P \in \mathfrak{B}$. A compactum is said to be $\mathfrak{B}$-like provided that for any $\varepsilon>0$ there is an onto $\varepsilon$-mapping from $X$ onto some $P \in \mathfrak{B}$.

First, we show the following

(3.1) THEOREM. Let $X$ be a continuum and let $\omega$ be any Whitney map for $\mathfrak{Q}=2^{X}$ or $C(X)$. Suppose that $t_{1}>t_{2}>\cdots$ is a decreasing sequence of positive numbers in $[0, \omega(X)]$ such that $t=\lim t_{n}$. If $\omega^{-1}\left(t_{n}\right)$ is weak $\mathfrak{B}$-like, then $\omega^{-1}(t)$ is also weak $\mathfrak{B}$-like.

For the case $\mathfrak{Q}=C(X)$, we have the following

(3.2) Corollary. The property of being weak $\mathfrak{B}$-like is a sequential strong Whitney-reversible property.

Note that $X$ is weak arc-like if and only if $X$ is arc-like (chainable), and $X$ is weak tree-like if and only if $X$ is tree-like. Hence by (3.2), we have the following which is an affirmative answer to [20, (14.57) and 21, (3.6)].

(3.3) COROLlaRY. The property of being chainable or tree-like is a sequential strong Whitney-reversible property.

To prove (3.1), we need the following simple lemma. For completeness, we give the proof.

(3.4) Lemma. Let $X$ be a subcompactum of a compactum $Y$. Let $f: X \rightarrow Z$ be an $\varepsilon$-mapping and let $\tilde{f}: Y \rightarrow Z$ be an extension of $f$. Then there is a closed neighborhood $U$ of $X$ in $Y$ such that $\tilde{f} \mid U: U \rightarrow Z$ is an $\varepsilon$-mapping. 
Proof. By the compactness of $X$, there is a positive number $\varepsilon_{1}$ such that $\varepsilon_{1}<\varepsilon$ and $f: X \rightarrow Z$ is an $\varepsilon_{1}$-mapping. Choose $\delta>0$ such that if $A \subset Z$ and $\operatorname{diam} A<\delta$, then $\operatorname{diam} f^{-1}(A)<\varepsilon_{1}$. Take $\delta^{\prime}>0$ such that if $x, y \in Y$ and $d(x, y)<\delta^{\prime}$, then $d(\tilde{f}(x), \tilde{f}(y))<\delta / 2$. Define a neighborhood $U$ of $X$ in $Y$ by letting

$$
U=\left\{y \in Y \mid d(y, x) \leqslant \min \left\{\left(\varepsilon-\varepsilon_{1}\right) / 2, \delta^{\prime}\right\} \text { for some } x \in X\right\} .
$$

We shall show that $\tilde{f} \mid U: U \rightarrow Z$ is an $\varepsilon$-mapping. Let $x, y \in U$ and $\tilde{f}(x)=\tilde{f}(y)$. Take points $x^{\prime}$ and $y^{\prime}$ of $X$ such that $d\left(x, x^{\prime}\right) \leqslant \delta^{\prime}$ and $d\left(y, y^{\prime}\right) \leqslant \delta^{\prime}$. Then we have

$$
d\left(f\left(x^{\prime}\right), f\left(y^{\prime}\right)\right) \leqslant d\left(\tilde{f}\left(x^{\prime}\right), \tilde{f}(x)\right)+d\left(\tilde{f}(y), \tilde{f}\left(y^{\prime}\right)\right)<\delta,
$$

which implies that $d\left(x^{\prime}, y^{\prime}\right)<\varepsilon_{1}$. Also, we have

$$
\begin{aligned}
d(x, y) & \leqslant d\left(x, x^{\prime}\right)+d\left(x^{\prime}, y^{\prime}\right)+d\left(y^{\prime}, y\right) \\
& <\left(\varepsilon-\varepsilon_{1}\right) / 2+\varepsilon_{1}+\left(\varepsilon-\varepsilon_{1}\right) / 2=\varepsilon .
\end{aligned}
$$

Hence $\tilde{f} \mid U: U \rightarrow Z$ is an $\varepsilon$-mapping.

Proof OF (3.1). Let $\varepsilon>0$. Assume that $M$ is an ANR that contains $\mathfrak{g}$. By (2.4), there is a positive number $\eta(\varepsilon / 3)$ such that if $t, t^{\prime} \in[0, \omega(X)]$ and $0 \leqslant t^{\prime}-t<$ $\eta(\varepsilon / 3)$, then $\omega^{-1}\left(t^{\prime}\right)$ is an $(\varepsilon / 3)$-approximate strong deformation retract of $\omega^{-1}\left(\left[t, t^{\prime}\right]\right)$ in $M$. Choose $t_{n}$ such that $t_{n}-t<\eta(\varepsilon / 3)$. Since $\omega^{-1}\left(t_{n}\right)$ is weak $\mathfrak{B}$-like, there is an ( $\varepsilon / 3$ )-mapping $f: \omega^{-1}\left(t_{n}\right) \rightarrow P$ for some $P \in \mathfrak{B}$. Since $P$ is an ANR, by (3.4) there is a neighborhood $U$ of $\omega^{-1}\left(t_{n}\right)$ in $M$ and there is an extension $\tilde{f}: U \rightarrow P$ of $f$ which is an $(\varepsilon / 3)$-mapping. Let $r: \omega^{-1}\left(\left[t, t_{n}\right]\right) \times[0, \infty) \rightarrow M$ be a mapping satisfying conditions $(*),(* *)$ and $(* * *)$. Choose a real $s$ in $[0, \infty)$ such that

$$
r\left(\omega^{-1}\left(\left[t, t_{n}\right]\right) \times[s, \infty)\right) \subset U .
$$

Set $g=r \mid \omega^{-1}(t) \times\{s\}: \omega^{-1}(t) \rightarrow U$. Now, we shall show that $\tilde{f} \circ g: \omega^{-1}(t) \rightarrow P$ is an $\varepsilon$-mapping. Let $A, B \in \omega^{-1}(t)$ and $\tilde{f} g(A)=\tilde{f} g(B)$. Since $\tilde{f}$ is an $(\varepsilon / 3)$-mapping, $d(g(A), g(B))<\varepsilon / 3$. Then we have

$$
\begin{aligned}
\rho_{\text {今) }}(A, B) & \leqslant d(A, g(A))+d(g(A), g(B))+d(g(B), B) \\
& <\varepsilon / 3+\varepsilon / 3+\varepsilon / 3=\varepsilon .
\end{aligned}
$$

Hence $\omega^{-1}(t)$ is weak $\mathfrak{B}$-like. This completes the proof.

Next, we shall prove the following

(3.5) Corollary. The property of being circle-like is a sequential strong Whitneyreversible property. More precisely, the property of being proper circle-like or chainable and circle-like is a sequential strong Whitney-reversible property.

To prove (3.5), we need the following definitions. A continuum $X$ is said to be $n$-decomposable provided that $X$ is the essential sum of $n$ continua, i.e., $X=X_{1} \cup$ $X_{2} \cup \cdots \cup X_{n}$, where $X_{i}$ is a subcontinuum of $X$ such that $X_{i}-\cup_{j \neq i} X_{j} \neq \varnothing$. A continuum $X$ is said to be $n$-indecomposable [2] provided that $X$ is $n$-decomposable 
and not $(n+1)$-decomposable. Clearly, $X$ is indecomposable if and only if $X$ is 1 -indecomposable.

(3.6) (C. E. BuRgess [2, THEOREM 7]). If $X$ is chainable, then in order that $X$ should be circle-like, it is necessary and sufficient that $X$ is either indecomposable or 2-indecomposable.

(3.7) Proposition. Let $X$ be $n$-decomposable and let $w$ be any Whitney map for $C(X)$. Then there is $t_{0} \in(0, \omega(X))$ such that if $t \leqslant t_{0}$, then $\omega^{-1}(t)$ is $n$-decomposable.

Proof. Let $X=A_{1} \cup A_{2} \cup \cdots \cup A_{n}$ be the essential sum of $n$ continua. Choose points $a_{i} \quad(i=1,2, \ldots, n)$ of $X$ such that $a_{i} \in A_{i}-\bigcup_{j \neq i} A_{j}$. Set $\varepsilon=$ $\min \left\{d\left(a_{i}, \cup_{j \neq i} A_{j}\right) \mid i=1,2, \ldots, n\right\}>0$. Choose $t_{0} \in(0, \omega(X))$ such that $A \in$ $\omega^{-1}\left(\left[0, t_{0}\right]\right)$, then diam $A<\varepsilon$. Consider the following sets in $\omega^{-1}(t)$ for $t \leqslant t_{0}$.

$$
X\left(A_{i}, \omega, t\right)=\left\{K \in \omega^{-1}(t) \mid K \cap A_{i} \neq \varnothing\right\} \quad(i=1,2, \ldots, n) .
$$

By $\left[14,3.2\right.$ Corollary], $X\left(A_{i}, \omega, t\right)$ is a subcontinuum of $\omega^{-1}(t)$. Then $\omega^{-1}(t)=$ $\bigcup_{i=1}^{n} X\left(A_{i}, \omega, t\right)$ is the essential sum of $n$ continua, which implies that $\omega^{-1}(t)$ is $n$-decomposable.

Proof of (3.5). By (3.2), we see that $X$ is chainable or circle-like. Since the property of being chainable is a Whitney property [10], the property of being proper circle-like is a sequential strong Whitney-reversible property. Now, we must show that the property of being chainable and circle-like is a sequential strong Whitneyreversible property. By (3.6), it is sufficient to prove that (a) the property of being indecomposable and chainable or (b) the property of being 2-indecomposable and chainable is a sequential strong Whitney-reversible property. By (3.3) and (3.7), the properties (a) and (b) are sequential strong Whitney-reversible properties. This completes the proof.

J. Krasinkiewicz [12, 3.3 Corollary], proved that if $X$ is circle-like, then for any Whitney map $\omega$ for $C(X), \operatorname{Sh}(X)=\operatorname{Sh}\left(\omega^{-1}(t)\right)$ for each $t \in[0, \omega(X))$. Hence, we have

(3.8) Corollary. Let $X$ be a continuum and let $\omega$ be any Whitney map for $C(X)$. Suppose that $t_{1}>t_{2}>\cdots$ is a decreasing sequence of positive numbers in $[0, \omega(X)]$ such that $\lim t_{n}=0$. If $\omega^{-1}\left(t_{n}\right)$ is circle-like, then $X$ is circle-like and $\operatorname{Sh}(X)=$ $\operatorname{Sh}\left(\omega^{-1}(t)\right)$ for each $0 \leqslant t<\omega(X)$.

(3.9) EXAMPLE. In the statement of (3.1), we cannot conclude that if $\omega^{-1}\left(t_{n}\right)$ is $\mathfrak{P}$-like, then $\omega^{-1}(t)$ is $\mathfrak{B}$-like in the case $\mathfrak{S}=2^{X}$. Consider the arc $X$ in the plane as below (see Figure 5). Let $\omega: 2^{X} \rightarrow[0, \omega(X)]$ be the Whitney map for $2^{X}$ as defined by [31] and the usual Euclidean metric. Then there is a decreasing sequence $t_{1}>t_{2}>\cdots$ of positive numbers in $[0, \omega(X)]$ such that $\lim t_{n}=0$ and $\omega^{-1}\left(t_{n}\right)$ is not connected. Hence there is a family $\mathfrak{P}$ of compact polyhedra such that each member of $\mathfrak{P}$ is not connected and $\omega^{-1}\left(t_{n}\right)$ is $\mathfrak{B}$-like $(n=1,2, \ldots)$ (see [17]). Since $X$ is connected, $X$ is not $\mathfrak{P}$-like. 


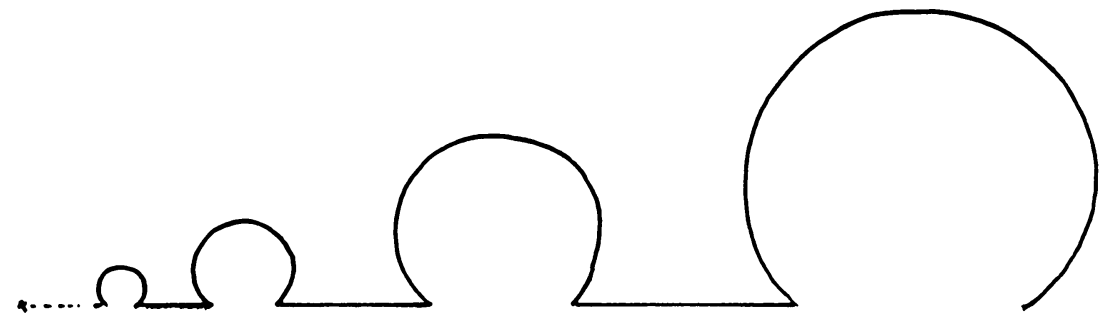

Figure 5

4. Inverse limits and approximations for Whitney maps. In this section, we consider the relation between inverse limits and Whitney maps.

In [22], A. Petrus showed that the property of being an FAR is not a Whitney property, more precisely, there exist a 2-dimensional FAR $X$ (in fact, $X=\mathrm{a}$ disk) and a Whitney map $\omega$ for $C(X)$ such that $\omega^{-1}(t)$ is not an FAR for some $t \in(0, \omega(X))$ (cf. (1.12)). Also, she proved that if $X$ is a dendrite (=1-dimensional $\mathrm{AR})$, then for any Whitney map $\omega$ for $C(X), \omega^{-1}(t)$ is contractible for each $t \in[0, \omega(X)]$. She had the following question [22]: Is the property of being contractible a Whitney property for 1 -dimensional continua? In relation to this question, we prove the following

(4.1) THEOREM. The property of being an FAR is a Whitney property for 1-dimensional continua.

To prove (4.1), we need the following approximation theorem for Whitney map.

(4.2) TheOREM (APPROXIMATION FOR WhitNey MAP). Let $\underline{X}=\left\{X_{n}, p_{n, n+1}\right\}$ be an inverse sequence of continua and let $X=\lim _{\leftarrow} \underline{X}$. Suppose that $\omega$ is a Whitney map for $\tilde{L}=2^{X}$ or $C(X)$. Then there exist Whitney maps $\omega_{n}(n \geqslant 1)$ for $\mathfrak{S}_{n}=2^{X_{n}}$ or $C\left(X_{n}\right)$ satisfying the conditions: for any $\varepsilon>0$, there is $n_{0}$ such that

$$
\begin{gathered}
d\left(\omega, \omega_{n} p_{n}^{*}\right)=\sup \left\{\left|\omega(A)-\omega_{n} p_{n}^{*}(A)\right| \mid A \in \mathfrak{Q}\right\}<\varepsilon \quad \text { and } \\
d\left(\omega_{m} p_{m, n}^{*}, \omega_{n}\right)<\varepsilon \quad \text { for each } n \geqslant m \geqslant n_{0},
\end{gathered}
$$

where $p_{n}: X \rightarrow X_{n}$ denotes the projection and $p_{n}^{*}: \mathfrak{S}_{\mathfrak{L}} \rightarrow \mathfrak{S}_{n}$ denotes the mapping defined by $p_{n}^{*}(A)=p_{n}(A)$ for $A \in \mathfrak{S}$.

Proof. Consider the Freudenthal space $\sigma X$ of $X$, i.e., $\sigma X$ is the set $X \cup \cup_{n=1}^{\infty} X_{n}$ with the topology defined by assuming that the totality of open subsets of the spaces $X_{n}$ and sets of the form $p_{n}^{-1}(U) \cup \cup_{m \geqslant n} p_{n, m}^{-1}(U)$, where $U$ is an open subset of $X_{n}$ $(n \geqslant 1)$, is an open base of $\sigma X$. Then $\sigma X$ is a compact metric space (e.g., see $J$. Krasinkiewicz, On a method of constructing ANR-sets. An application of inverse limits, Fund. Math. 92 (1976), p. 98). Assume that $\sigma X$ is lying in the Hilbert cube $Q$. By (1.2), there is a Whitney map $\mu: \mathfrak{S}^{\prime} \rightarrow[0, \mu(Q)] \hookrightarrow[0, \infty)$ for $\mathfrak{Q}^{\prime}=2^{Q}$ or $C(Q)$, which is an extension of $\omega$. Set $\omega_{n}=\mu \mid \mathfrak{S}_{n}$, where $\mathfrak{S}_{n}=2^{X_{n}}$ or $C\left(X_{n}\right)$. Note that for 
any $\varepsilon^{\prime}>0$, there is $n_{1}$ such that if $n \geqslant m \geqslant n_{1}$, then $d\left(i_{X}, p_{n}\right)<\varepsilon^{\prime}$ and $d\left(i_{X_{n}}, p_{m, n}\right)$ $<\varepsilon^{\prime}$, where $i_{X}: X \rightarrow X \hookrightarrow \sigma X$ is the natural inclusion and $p_{n}: X \rightarrow X_{n} \hookrightarrow \sigma X$, etc. By using this fact and the continuity of $\mu$, we can easily see that there exist $n_{0}$ satisfying the desired conditions.

Proof of (4.1). Since $X$ is tree-like, there is an inverse sequence $\underline{X}=\left\{X_{n} P_{n, n+1}\right\}$ such that each $X_{n}$ is a tree and $X=\lim _{\leftarrow} \underline{X}$. Let $\varepsilon_{1}>\varepsilon_{2}>\varepsilon_{3}>\cdots$ be a decreasing sequence of positive numbers such that $\sum \varepsilon_{i}<\infty$. By (4.2), without loss of generality, we may assume that there are Whitney maps $\omega_{n}$ for $C\left(X_{n}\right)$ such that

$$
d\left(\omega, \omega_{n} p_{n}^{*}\right)<\varepsilon_{n} \text { and } d\left(\omega_{n} p_{n, m}^{*}, \omega_{m}\right)<\varepsilon_{n} \text { for } m \geqslant n .
$$

Let $t \in(0, \omega(X))$. Let $A \in \omega_{n+1}^{-1}\left(\left[t-\sum_{i=n+1}^{\infty} \varepsilon_{i}, t+\sum_{i=n+1}^{\infty} \varepsilon_{i}\right]\right)$. Then we have

$$
\begin{aligned}
\left|\omega_{n} p_{n, n+1}^{*}(A)-t\right| & \leqslant\left|\omega_{n} p_{n, n+1}^{*}(A)-\omega_{n+1}(A)\right|+\left|\omega_{n+1}(A)-t\right| \\
& \leqslant \varepsilon_{n}+\sum_{i=n+1}^{\infty} \varepsilon_{i}=\sum_{i=n}^{\infty} \varepsilon_{i} .
\end{aligned}
$$

Hence

$$
p_{n, n+1}^{*}(A) \in \omega^{-1}\left(\left[t-\sum_{i=n}^{\infty} \varepsilon_{i}, t+\sum_{i=n}^{\infty} \varepsilon_{i}\right]\right) .
$$

This implies that

$$
p_{n, n+1}^{*} \omega_{n+1}^{-1}\left(\left[t-\sum_{i=n+1}^{\infty} \varepsilon_{i}, t+\sum_{i=n+1}^{\infty} \varepsilon_{i}\right]\right) \subset \omega_{n}^{-1}\left(\left[t-\sum_{i=n}^{\infty} \varepsilon_{i}, t+\sum_{i=n}^{\infty} \varepsilon_{i}\right]\right) .
$$

Consider the following inverse sequence $\omega^{-1}(t)$;

$$
\omega_{1}^{-1}\left(\left[t-\alpha_{1}, t+\alpha_{1}\right]\right) \stackrel{p_{12}^{*}}{\leftarrow} \omega_{2}^{-1}\left(\left[t-\alpha_{2}, t+\alpha_{2}\right]\right) \stackrel{p_{23}^{*}}{\leftarrow} \omega_{3}^{-1}\left(\left[t-\alpha_{3}, t+\alpha_{3}\right]\right) \leftarrow,
$$

where $\alpha_{n}=\sum_{i=n}^{\infty} \varepsilon_{i}$.

Let $A \in \omega^{-1}(t)$. Then we have

$$
\left|\omega_{n} p_{n}^{*}(A)-t\right|=\left|\omega_{n} p_{n}^{*}(A)-\omega(A)\right|<\varepsilon_{n},
$$

which implies that

$$
p_{n}^{*}\left(\omega^{-1}(t)\right) \subset \omega_{n}^{-1}\left(\left[t-\varepsilon_{n}, t+\varepsilon_{n}\right]\right) \subset \omega^{-1}\left(\left[t-\alpha_{n}, t+\alpha_{n}\right]\right) .
$$

Let $\left(A_{n}\right)_{n} \in \lim _{\leftarrow} \omega^{-1}(t)$. Set $A=\lim _{\leftarrow}\left\{A_{n}, p_{n, n+1} \mid A_{n+1}\right\}$. Then $A \in C(X)$. Also,

$$
\begin{aligned}
|\omega(A)-t| & \leqslant \mid \omega\left(\overleftarrow{A)}-\omega_{n} p_{n}^{*}(A)|+| \omega_{n} p_{n}^{*}(A)-t \mid\right. \\
& <\varepsilon_{n}+\alpha_{n} \quad \text { for each } n .
\end{aligned}
$$

If $n \rightarrow \infty$, then $\varepsilon_{n}+\alpha_{n} \rightarrow 0$. Hence $\omega(A)=t$. Therefore, we conclude that $\omega^{-1}(t)=$ $\lim _{\leftarrow} \omega^{-1}(t)$. Since each $X_{n}$ is a tree, by the proof of [22, Proposition 12 or 5, (2.17)], we can see that $\omega_{n}^{-1}\left(\left[t-\alpha_{n}, t+\alpha_{n}\right]\right)$ is an AR for each $n=1,2, \ldots$ Hence $\omega^{-1}(t)$ is an FAR. This completes the proof.

5. Problems. In this section, we give the following problems.

(5.1) Is it true that the movability is a Whitney property?

(5.2) Is it true that the property of being pointed 1-movable, or movable is a (sequential) strong Whitney-reversible property?

(5.3) Is it true that the property of being $\mathfrak{B}$-like is a sequential strong Whitneyreversible property? 
(5.4) Is the property of being an FANR a Whitney property for 1-dimensional continua?

ADDED IN PROOF. Recently, the author proved that (5.1) has a negative answer.

\section{REFERENCES}

1. K. Borsuk, Theory of shape, Monograf. Mat. 59 (1975).

2. C. E. Burges, Chainable continua and indecomposability, Pacific J. Math. 9 (1959(), 653-659.

3. D. Dydak, A simple proof that pointed connected FANR-spaces are regular fundamental retracts of ANRs, Bull. Acad. Polon. Sci. Sér. Sci. Math. 25 (1977), 55-62.

4. J. Dydak and J. Segal, Strong shape theory, Dissertations Math. 191 (1982), 1-42.

5. J. T. Goodykoontz, Jr. and S. B. Nadler, Jr., Whitney levels in hyperspaces of certain Peano continua, Trans. Amer. Math. Soc. 274 (1982), 671-694.

6. H. Kato, Concerning hyperspaces of certain peano continua and strong regularity of Whitney maps, Pacific J. Math. 119 (1985), 159-167.

7. J. L. Kelley, Hyperspaces of a continuum, Trans. Amer. Math. Soc. 52 (1942), 22-36.

8. Y. Kodama and J. Ono, On fine shape theory. I, II, Fund. Math. 105 (1979), 29-39; 108 (1980), $89-98$.

9. A. Koyama, A note on some strong Whitney-reversible properties, Tsukuba J. Math. 4 (1980), $313-316$.

10. J. Krasinkiewicz, On the hyperspaces of snake-like and circle-like continua, Fund. Math. 83 (1974), $155-164$.

11. On the hyperspaces of hereditarily indecomposable continua, Fund. Math. 84 (1974), $175-186$.

12. Shape properties of hyperspaces, Fund. Math. 101 (1978), 79-91.

13. J. Krasinkiewicz and P. Minc, Generalized paths and pointed 1-movability, Fund. Math. 104 (1979), $141-53$.

14. J. Krasinkiewicz and S. B. Nadler, Jr., Whitney properties, Fund. Math. 98 (1978), 165-180.

15. A. Y. Lau, Whitney continuum in hyperspace, Topology Proc. 1 (1976), 187-189.

16. W. Lewis, Continuum theory problems, Topology Proc. 8 (1983), 361-394.

17. S. Mardešić and J. Segal, $\varepsilon$-mappings onto polyhedra, Trans. Amer. Math. Soc. 109 (1963), 146-164.

18. __ Shape theory, North-Holland Mathematical Library, North-Holland, Amsterdam, 1982.

19. S. B. Nadler. Jr., A characterization of locally connected continua by hyperspaces retractions, Proc. Amer. Math. Soc. 67 (1977), 167-176.

20. __ Hyperspaces of sets, Pure and Appl. Math., vol. 49, Dekker, New York, 1978.

21. Whitney-reversible properties, Fund. Math. 109 (1980), 235-248.

22. A. Petrus, Contractibility of Whitney continua in $C(X)$, General Topology and Appl. 9 (1978), $275-288$.

23. J. T. Rogers, Jr., The cone $=$ hyperspace property, Canad. J. Math. 24 (1972), 279-285.

24. Continua with cones homeomorphic to hyperspaces, General Topology and Appl. 3 (1973), $283-289$

25. Dimension and the Whitney subcontinua of $C(X)$, General Topology and Appl. 6 (1976), $91-100$

26. __ Dimension of hyperspaces, Bull. Acad. Polon. Sci. Sér. Sci. Math. 20 (1972), 177-179.

27. Embedding the hyperspaces of circle-like plane continua, Proc. Amer. Math. Soc. 29 (1971), $165-168$.

28. . Hyperspaces of arc-like and circle-like continua, Lecture Notes in Math., vol. 375, Springer-Verlag, New York, 1974, pp. 213-234.

29. Whitney continua in the hyperspaces $C(X)$, Pacific J. Math. 58 (1975), 569-584.

30. L. E. Ward, Jr., Extending Whitney maps, Pacific J. Math. 93 (1981), 465-469.

31. H. Whitney, Regular families of curves. I, Proc. Nat. Acad. Sci. U.S.A. 18 (1932), 275-278.

32. M. Wojdyslawski, Retracte absolus et hyperspaces des continus, Fund. Math. 32 (1939), 184-192.

33. E. Abo-Zeid, Some results in continua theory and hyperspaces, Dissertation, Univ. of Saskatchewan, Saskatoon, Canada.

\section{InStITUTE: of Mathematics, University OF TSUKUba, Ibaraki 305 JaPan}

Current address: Faculty of Integrated Arts and Sciences, Hiroshima University, Higashisenda-Machi, Naka-ku. Hiroshima 730, Japan 